Pacific Journal of Mathematic 


\title{
RELATIONS NOT DETERMINING THE STRUCTURE OF $L$
}

\author{
JOHN ROSENTHAL
}

\begin{abstract}
A relation $S$ is said to be determined up to isomorphism by relations $R$ with respect to a theory $K$ if for all models $\mathfrak{U}_{1}, \mathfrak{A}_{2}$ of $K, \mathfrak{U}_{1}$ restricted to $R$ is isomorphic to $\mathfrak{U}_{2}$ restricted to $R$ implies $\mathfrak{A}_{1}$ is isomorphic to $\mathfrak{A}_{2}$. In this paper simple necessary conditions for $S$ to be determined up to isomorphism by $R$ are given. These are applied in set theory to show there are (nonstandard) models of set theory with isomorphic ordinals and nonisomorphic constructible sets. The isomorphism on the ordinals may be taken to preserve many familiar arithmetic functions on the ordinals as addition, multiplication and exponentiation.
\end{abstract}

In this paper we show that the structure of the constructible sets of a model of set theory is not determined by the order-type of its ordinals, or, in fact, by its ordinals with various familiar arithmetic functions. This is shown by exhibiting (nonstandard) models of set theory with isomorphic ordinals and nonisomorphic constructible sets. The isomorphism on the ordinals may be taken to preserve familiar arithmetic functions.

These results are obtained by the use of certain simple general model-theoretic results developed in $\S 2$. We define a relation $S$ to be determined up to isomorphism by a set of relations $R$ with respect to a theory $K$ if $\left\langle A, R_{A}, S_{A}\right\rangle \vDash K,\left\langle B, R_{B}, S_{B}\right\rangle \vDash K,\left\langle A, R_{A}\right\rangle \approx\left\langle B, R_{B}\right\rangle$ implies $\left\langle A, R_{A}, S_{A}\right\rangle \approx\left\langle B, R_{B}, S_{B}\right\rangle$. We then give two simple sufficient conditions for $S$ not to be determined up to isomorphism by $R$ wrt $K$. Firstly, by a modification of a model-theoretic proof of Beth's theorem relating implicit and explicit definability, we show $S$ is not determined up to isomorphism by $R$ if there is a sentence $\sigma$ such that the consequences about $R$ of $K, K \cup\{\sigma\}, K \cup\{\neg \sigma\}$ are all the same. Using this, we show $S$ is not determined up to isomorphism by $R$ wrt $K$ if there is a model $\mathfrak{U}$ of $K$ in which the truth set of $\mathfrak{A}$ is not Turing-reducible to $K$ join the truth set of $\mathfrak{U}$ restricted to $R$.

After illustrating simple applications of these results in $\S 2$, we turn to the main set theory results in $\S 3$. We observe that for any model $\mathfrak{U}$ of set theory, $\left\langle O n_{\mathfrak{Q}},<_{\mathfrak{A}}\right\rangle \equiv\left\langle\omega^{\omega},\langle\rangle\right.$ which has recursive truth set [9] and that the truth set of $\left\langle O n_{\mathfrak{A}},<_{\mathfrak{Q}}, \tilde{\varepsilon}_{\mathfrak{A}}, \cong_{\mathfrak{A}}\right\rangle$ is not recursive (where $F$ is the map defined up Gödel from $O n \rightarrow L, \alpha \tilde{\varepsilon} \beta$ if $F(\alpha) \varepsilon F(\beta), \alpha \cong \beta$ if $F(\alpha)=F(\beta)$ [8;16]. Using this we may by 
our model-theoretic results immediately construct models of set theory with isomorphic ordinals and nonisomorphic constructible sets. This result is extended to include various functions on the ordinals by generalizing the above approach.

2. Notation and model theory results. Our notation will generally follow that suggested in [1] with the following additions and modifications: Capital italic letters will denote either relations and operations, or sets of relations and operations. Capital italic letters will also denote either relation and operation symbols, or sets of relations and operation symbols. Of course, we will use certain standard relation or operation symbols such as $<,+, \times$ etc.

Say $A$ is a set of operation and relation symbols. Then $\Sigma(A)$ is the sentences of the first-order language with relation and operations symbols the set $A$. We let $T h_{\mathfrak{Z}}$ be the set of all sentences true of $\mathfrak{U}$ in the object language of $\mathfrak{X}$. We let $\operatorname{Tr}_{\mathfrak{X}}(A)$ be $T h_{\mathfrak{X}} \cap \Sigma(A)$. Finally $C n(K)=\{\sigma \mid K \vDash \sigma\}$. For certain special subsets of $\Sigma(A)$ we use standard symbols, e.g., $Z F$ for the axioms of Zermelo-Fraenkel set theory.

In general given an $\omega$-enumeration of $A$, a set of relation and operation symbols, we use this to define godel numbers of elements of $\Sigma(A)$. We denote the godel numbers of sentences by the sentences themselves, and similarly the set of godel numbers of a set of sentences by the set of sentences.

Let $K$ be a theory in $\Sigma(A, B)$ (where $A, B$ are sets of relation and operation symbols). We say $B$ is determined up to $\approx$ by $A$ with respect to (wrt) $K$ if for every models $\left\langle C_{1}, A_{1}, B_{1}\right\rangle,\left\langle C_{2}, A_{2}, B_{2}\right\rangle \vDash K$, $\left\langle C_{1}, A_{1}\right\rangle \approx\left\langle C_{2}, A_{2}\right\rangle$ implies $\left\langle C_{1}, A_{1}, B_{1}\right\rangle \approx\left\langle C_{2}, A_{2}, B_{2}\right\rangle$ (or equivalently if for every models $\left.\langle C, A, B\rangle,\left\langle C, A, B^{\prime}\right\rangle \vDash K,\langle C, A, B\rangle \approx\left\langle C, A, B^{\prime}\right\rangle\right)$. We say $B$ is determined up to $\equiv$ by $A$ with respect to $K$ if for every models $\left\langle C_{1}, A_{1}, B_{1}\right\rangle,\left\langle C_{2}, A_{2}, B_{2}\right\rangle \vDash K,\left\langle C_{1}, A_{1}\right\rangle \approx\left\langle C_{2}, A_{2}\right\rangle$ implies $\left\langle C_{1}, A_{1}, B_{1}\right\rangle \equiv\left\langle C_{2}, A_{2}, B_{2}\right\rangle$. In general we omit mention of $K$ when it is clear.

Trivially, $B$ is not determined up to $\equiv$ by $A$ implies $B$ is not determined up to $\approx$ by $A$.

Let $K$ be a theory in $\Sigma(A)$. We say $K$ is complete wrt $\Sigma^{\prime} \leqq \Sigma(A)$ if for every $\sigma \in \Sigma^{\prime}, K \vDash \sigma$ or $K \vDash \neg \sigma$.

THEOREM 1. The following 4 are equivalent

(a) For every model $\mathfrak{A}$ of $K, K \cup \operatorname{Tr}_{\mathfrak{Q}}(A)$ is complete wrt $\Sigma(A, B)$.

(b) For every $\sigma \in \Sigma(A, B)$, there is a $\tau \in \Sigma(A)$ such that

$$
K \vDash(\sigma \leftrightarrow \tau) \text {. }
$$

(c) $B$ is determined up to $\equiv$ by $A$ wrt $K$. 
(d) For all models $\left\langle C_{1}, A_{1}, B_{1}\right\rangle$ and $\left\langle C_{2}, A_{2}, B_{2}\right\rangle$ of $K$,

$$
\left\langle C_{1}, A_{1}\right\rangle \equiv\left\langle C_{2}, A_{2}\right\rangle \rightarrow\left\langle C_{1}, A_{1}, B_{1}\right\rangle \equiv\left\langle C_{2}, A_{2}, B_{2}\right\rangle \text {. }
$$

Proof. We show $(\mathrm{b}) \Rightarrow(\mathrm{a}) \Rightarrow(\mathrm{d}) \Rightarrow(\mathrm{c}) \Rightarrow(\mathrm{b})$. The first three implications are trivial and left to the reader. $(\mathrm{c}) \Rightarrow(\mathrm{b})$ is a routine application of the Robinson Joint Consistency Theorem [11]. Assume $(\exists \sigma \varepsilon \Sigma(A, B))(\forall \tau \varepsilon \Sigma(A))(\neg K \models(\sigma \leftrightarrow \tau))$. Let $\sigma_{0}$ be such a $\sigma$. So $K \cup$ $\left\{\sigma_{0}\right\}, K \cup\left\{\neg \sigma_{0}\right\}$ are consistent and $\neg(\exists \tau \varepsilon \Sigma(A))\left(\tau \varepsilon C n\left(K \cup\left\{\sigma_{0}\right\}\right)\right.$ and $\left.(\neg \tau) \varepsilon C n\left(K \cup\left\{\neg \sigma_{0}\right\}\right)\right)$.

Relabel the symbols of $B$ preserving arity so as to be symbols not in $A \cup B$. Call this new set $B^{\prime}$. Let $\phi^{\prime}$ in general be $\varphi$ with symbols in $B$ replaced by the corresponding ones in $B^{\prime}$. So

$$
\neg(\exists \tau \varepsilon \Sigma(A))\left(\tau \varepsilon C n\left(K \cup\left\{\sigma_{0}\right\}\right) \text { and }(\neg \tau) \varepsilon C n\left(K^{\prime} \cup\left\{\neg \sigma_{0}^{\prime}\right\}\right)\right),
$$

i.e., the hypotheses of the joint consistency theorem. So there is a $\mathfrak{c}=\left\langle C, A, B, B^{\prime}\right\rangle$ such that $\mathfrak{\complement} \vDash K \cup\left\{\sigma_{0}\right\} \cup K^{\prime} \cup\left\{\neg \sigma_{0}^{\prime}\right\}$. So $\langle C, A, B\rangle \vDash$ $K \cup\left\{\sigma_{0}\right\},\left\langle C, A, B^{\prime}\right\rangle \vDash K \cup\left\{\neg \sigma_{0}\right\}$. So

$$
\langle C, A, B\rangle \not \equiv\left\langle C, A, B^{\prime}\right\rangle,
$$

i.e., $B$ is not determined up to $\equiv$ by $A$.

Let $K$ be a theory in $\Sigma(A, B)$. We say $B$ is Turing determined by $A$ wrt $K$ if for every model $\mathfrak{i}$ of $K, \operatorname{Tr}_{\mathfrak{Q}}(A, B) \leqq_{T} K$ join $\operatorname{Tr}_{\mathfrak{Q}}(A)$. Note-we are here assuming $\omega$-enumerations of $A, B$ have been given and that the derived godel numbers of sentences are abusively denoted by the sentences themselves. Also if $K$ is recursive, then this is equivalent to $\operatorname{Tr}_{\mathfrak{2 i}}(A, B) \leqq_{T} \operatorname{Tr}_{\mathfrak{Y I}}(A)$. ( $\leqq_{T}$ is Turing reducible.)

Theorem 2. $B$ is determined up to $\equiv$ by $A$ wrt $K$ implies $B$ is Turing determined by $A$ wrt $K$.

Proof. By Theorem 1 , if $B$ is determined up to $\equiv$ by $A$, then $K \cup \operatorname{Tr}_{\mathfrak{N}}(A)$ is complete wrt $\Sigma(A, B)$ for every model $\mathfrak{i}$ of $K$. But hence $\operatorname{Tr}_{\mathfrak{U}}(A, B) \leqq_{T} K$ join $\operatorname{Tr}_{\mathfrak{U}}(A)$.

Remark. We see, thus, that $B$ Turing determined by $A$ wrt $K \rightarrow$ $B$ determined up to $\equiv$ by $A$ wrt $K \rightarrow B$ determined up to $\approx$ by $A$ wrt $K \rightarrow B$ determined up to $=$ by $A$ wrt $K$ (i.e., $B$ is implicitly definable in terms of $A$ wrt $K$ ). The converses of these do not hold. We give examples

(1) Let $F, G$ be unary relation symbols. Let $K \cong \Sigma(F, G)$ be 
$\{\exists ! x F(x), \exists ! x G(x), \neg(F(x) \wedge G(x))\}$. Then $G$ is not determined up to $=$ by $F$ wrt $K$. But $G$ is determined up to $\approx$ by $F$ wrt $K$.

(2) Let $<$ be a binary relation symbol, $F$ be a unary relation symbol. Let $K \cong \Sigma(<, F)$ be $T h\langle Q,\langle, 0\rangle$.

Then $F$ is not determined up to $\approx$ by $<$ wrt $K$.

$$
\begin{aligned}
\text { (e.g., }\langle(Q \cap(-\infty, 0)) \cup(R \cap[0, \infty)),<,-1\rangle \\
\quad \neq\langle(Q \cap(-\infty, 0)) \cup(R \cap[0, \infty)),<, 1\rangle .)
\end{aligned}
$$

But $F$ is determined up to $\equiv$ by $<$ wrt $K$ (as $K$ complete).

(3) Let $<$ be a binary relation symbol, $F$ a unary relation symbol. Let $K \cong \Sigma(<, F)$ be theory of dense linear order without endpoints $\cup\{\exists x \exists y(F(x) \wedge F(y) \wedge(\forall z)(F(z) \rightarrow x=z \vee y=z))\}$. Then $F$ is not determined up to $\equiv$ by $<$ wrt $K$ (as $\exists ! x F(x)$ is undecided). Yet in any model $\mathfrak{N}: \operatorname{Tr}_{\mathfrak{A}}(<, F)$ is recursive and hence $\leqq_{T} \operatorname{Tr}_{\mathfrak{A}}(<)$. So $F$ is Turing determined by $<\operatorname{wrt} K$.

REMARK. We give several simple well-known examples illustrating the use of Theorem 2.

(1) Let $K=\mathrm{ENT}$ (elementary number theory). Then $\times$ is not determined up to $\equiv$ by $<$, + .

Proof. Let $\mathfrak{N}=\langle\omega,<,+, \times\rangle$. So $T_{1}=\operatorname{Tr}_{\mathfrak{N}}(<,+)$ is recursive, $T_{2}=T h_{\mathfrak{R}}$ is not. So $T_{2} \varlimsup_{T} T_{1}$. So by Theorem $2, \times$ is not determined up to $\equiv$ by $<$, + . In fact, this result also holds for $K=$ any other arithmetic set of formulae true about $\mathfrak{R}$. Similar results hold for arithmetic theories with $\mathfrak{\Omega}=\langle Q,\langle,+, \times\rangle$ as a model.

(2) Add to the standard symbols $(<,+, \times, 0,1)$ of real closed fields an additional unary relation symbol $i$ which is intended just to apply to integers.

Let $K=\mathrm{RCF}$ (the theory of real closed fields)

$$
\begin{aligned}
\cup\{i(0), i(x) \rightarrow i(x+1) \wedge i(x-1), & \neg(\exists x)(0<x<1 \wedge i(x)), \\
& \neg(\exists x)(\forall y)(x \leqq y \leqq x+1 \rightarrow \neg i(y))\}
\end{aligned}
$$

$\cup$ any arithmetic set of formulae true about $\mathfrak{R}$ relativized to

$$
x \geqq 0 \wedge i(x) \text {. }
$$

Then $i$ is not determined up to $\approx$ by $<,+, \times, 0,1$.

Proof. As if $\Re=\langle R,<,+, \times, i\rangle$, then $\operatorname{Tr}_{\mathfrak{R}}(<,+, \times)$ join $K$ is arithmetic, but $T h_{\Re}$ is not and hence not $\leqq_{T} \operatorname{Tr}_{\Re}(<,+, \times)$ join $K$. 
Similar results are obtained for algebraically closed fields.

3. Applications to set theory. We show in this chapter that the structure of the constructible sets is not determined up to isomorphism by the order type of the ordinals, nor in fact, by certain fairly large classes of functions on the ordinals. We proceed as follows:

Let $F$ be the map of $O n$ (the ordinals) onto $L$ (the constructible sets) defined by Godel [8]. Let

$$
\tilde{\varepsilon}(\alpha, \beta)={ }_{d f} F(\alpha) \varepsilon F(\beta) ; \cong(\alpha, \beta)={ }_{d f} F(\alpha)=F(\beta) .
$$

Takeuti defines [16] a theory of primitive recursive functions and relations on the ordinals, which we will call ONT (Ordinal number theory). In particular he shows:

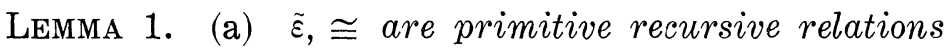

(b) if $\langle O n,\langle, P R\rangle \vDash \mathrm{ONT}$, then $\langle O n, \tilde{\varepsilon}$, $\cong \vDash \vDash Z F, V=L$ and $\left\langle O n,\langle, P R\rangle \approx\left\langle O n_{\langle 0 n, \tilde{\varepsilon}, \cong\rangle},\left\langle\langle 0 n, \tilde{\varepsilon}, \cong\rangle, P R_{\langle 0 n, \tilde{\varepsilon}, \cong\rangle}\right\rangle\right.\right.$

(c) if $\mathfrak{U} \vDash Z F$, then $\left\langle O n_{\mathfrak{Y}},<_{\mathfrak{U}}, P R_{\mathfrak{U}}\right\rangle \vDash \mathrm{ONT}$ (if $\mathfrak{U} \vDash Z F$, then $O n_{\mathfrak{A}}$ are its ordinals, $<_{\mathfrak{A}}$ the $<$ relation on its ordinals and $P R_{\mathfrak{U}}$ the primitive recursive functions on the ordinals.)

Furthermore $\cong$ is definable in terms of $\tilde{\varepsilon}$.

Proofs. Omitted.

So if $\tilde{\varepsilon}$ is not determined up to $\approx$ (or $\equiv$ ) by $<$ (i.e., if there are models $\left\langle O n,\left\langle, P R_{1}\right\rangle,\left\langle O n,<, P R_{2}\right\rangle \vDash O N T\right.$ such that $\left\langle O n,<, \tilde{\varepsilon}_{1}\right\rangle \nsim$ (or $\not \equiv)\left\langle O n,\left\langle, \tilde{\varepsilon}_{2}\right\rangle\right.$ ); then by Lemma $1 b \mathfrak{U}_{i}=\left\langle O n, \tilde{\varepsilon}_{i}, \cong_{i}\right\rangle \vDash Z F, V=L$. Furthermore by Lemma $1 b,\left\langle O n_{\mathfrak{H}_{i}},<_{\mathfrak{H}_{i}}\right\rangle \approx\langle O n,\langle\rangle$ and hence

$$
\left\langle O n_{\mathfrak{H}_{1}},<_{\mathfrak{A}_{1}}\right\rangle \approx\left\langle O n_{\mathfrak{H}_{2}},<_{\mathfrak{H}_{2}}\right\rangle \text {. }
$$

But by assumption $\left\langle O n, \tilde{\varepsilon}_{1}\right\rangle \approx$ (or $\left.\not \equiv\right)\left\langle O n, \tilde{\varepsilon}_{2}\right\rangle$, and so $\mathfrak{N}_{1} \neq$ (or $\left.\not \equiv\right) \mathfrak{N}_{2}$. As $\mathfrak{I}_{i} \vDash V=L, \mathfrak{N}_{i}=\left\langle L_{\mathfrak{A} i}, \varepsilon_{\mathfrak{Q}}\right\rangle$. So there are models $\mathfrak{N}_{1}, \mathfrak{T}_{2} \vDash Z F$ such

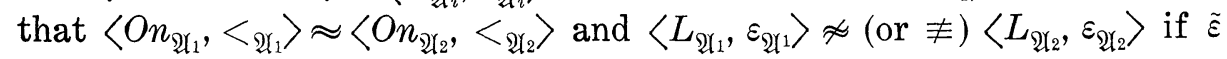
is not determined up to $\approx$ (or $\equiv$ ) by $<$.

We usually will show $\tilde{\varepsilon}$ is not determined up to $\equiv$ by $<$ by showing it is, in fact, not Turing determined by $<$. Similarly and more generally we have

Proposition 2. If $\tilde{\varepsilon}$ is not Turing determined by $<$ and some class of primitive recursive functions, then the structure of $L$ is not determined up to $\equiv$ by the strusture of the ordinals with that class of primitive recursive functions on them. 
Let $\mathfrak{Y}$ be any arbitrary model of $Z F, V=L$. Then as is well known $T h_{\mathfrak{A}}$ is not recursive. As $\mathfrak{X} \vDash V=L$,

$$
\mathfrak{U} \approx\left\langle L_{\mathfrak{Q}}, \varepsilon_{\mathfrak{A}}\right\rangle \approx\left\langle O n_{\mathfrak{A}}, \tilde{\varepsilon}_{\mathfrak{A}}, \cong_{\mathfrak{U}}\right\rangle .
$$

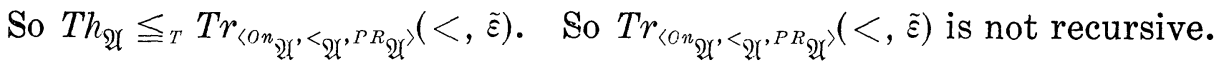

THEOREM 3. $\operatorname{Tr}_{\left\langle{ }^{\prime} n,<_{\mathfrak{H}}, P R_{\mathfrak{H}}\right\rangle}(<)$ is recursive.

Proof. Omitted. We recommend the interested reader examine the proof in [7].

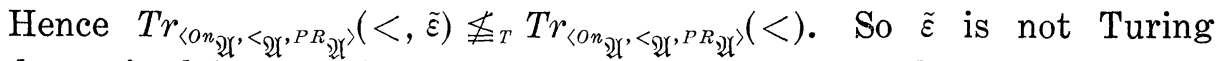
determined by $<$. So by the above arguments we have

COROLlaRY 4. If $Z F$ is consistent, then there are models $\mathfrak{N}, \mathfrak{Y} \mathfrak{X}^{\prime} \vDash$

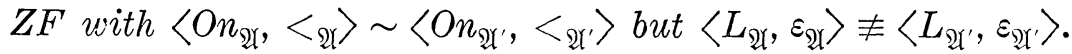

To extend this result to preserve various functions on the ordinals in the isomorphism, we need results similar to Theorem 3 in order to conclude $\neg\left(\operatorname{Tr}_{\left\langle 0 n_{\mathfrak{Q}},<_{\mathfrak{U}}, P R_{\mathfrak{U}}\right\rangle}(<, A, \tilde{\varepsilon}) \leqq_{T} \operatorname{Tr}_{\left\langle 0 n_{\mathfrak{Q}},<_{\mathfrak{A}}, P R_{\mathfrak{H}}\right\rangle}(<, A)\right)$ for appropriate classes $A$ of primitive recursive functions. Also we will wish to consider nonprimitive-recursive functions in $A$. So we will have to expand $P R_{\mathfrak{Y}}$, i.e., we will have to change it to $\mathrm{ARITH}_{\mathfrak{Y}}$, the arithmetic functions on the ordinals of $\mathfrak{U}$ which we will shortly define.

Theorem 3 will be extended by application of

LEMMA 5. Let $A$ be a set of primitive recursive functions.

If there is a model $\mathfrak{U}$ of $Z F$ and an ordinal $\alpha_{\mathfrak{Z}} \varepsilon O n_{\mathfrak{Z}}$ sush that

1. $\left\langle O n_{\mathfrak{Y}},<_{\mathfrak{W}}, A_{\mathfrak{H}}\right\rangle \equiv\left\langle\alpha_{\mathfrak{Y}},<_{\mathfrak{H}}, A_{\mathfrak{A}}\right\rangle$ and

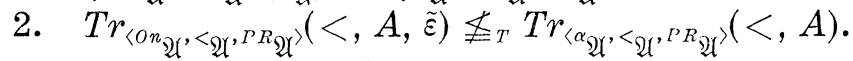

then the structure of $L$ is not determined up to $\equiv$ by the strusture of the ordinals with the class $A$ of primitive recursive functions on them.

Proof. For by 1, 2 above we have $\tilde{\varepsilon}$ is not Turing determined by $A$. So by Proposition 2 we are done.

We will show results of the form $\left\langle O n_{\mathfrak{Y}},\left\langle_{\mathfrak{Y}}, A_{\mathfrak{Y}}\right\rangle \equiv\left\langle\alpha_{\mathfrak{Y}},<_{\mathfrak{Q}}, A_{\mathfrak{Y}}\right\rangle\right.$ by use of a technique of Ehrenfeucht [7]. Given models $\mathfrak{V}_{1}, \mathfrak{U}_{2}$ he defines 2 person finite games $G_{n}\left(\mathfrak{I}_{1}, \mathfrak{A}_{2}\right), H_{n}\left(\mathfrak{A}_{1}, \mathfrak{A}_{2}\right)$ and shows that if for all $n$ the second player has a winning strategy in $G_{n}\left(\mathfrak{H}_{1}, \mathfrak{A}_{2}\right)$ then $\mathfrak{A}_{1} \equiv \mathfrak{A}_{2}$. A similar slightly stronger result holds for $H_{n}\left(\mathfrak{A}_{1}, \mathfrak{A}_{2}\right)$. So to generalize Corollary 4 we must merely by the above argument meet the hypothesis of the following lemma: 
Lemma 6. Let $A$ be a set of primitive recursive functions. If there is a model $\mathfrak{X}$ of $Z F$ and an ordinal $\alpha_{\mathfrak{H}} \varepsilon O n_{\mathfrak{U}}$ such that 1. Player II has a winning strategy in

$$
H_{n}\left(\left\langle O n_{\mathfrak{Y}},<_{\mathfrak{A}}, A_{\mathfrak{U}}\right\rangle,\left\langle\alpha_{\mathfrak{A}},<_{\mathfrak{I}}, A_{\mathfrak{U}}\right\rangle\right), \forall n
$$

2. As in Lemma 5, then the same conclusions as in Lemma 5 hold.

In particular, Ehrenfeucht has shown by these techniques that THEOREM 7 .

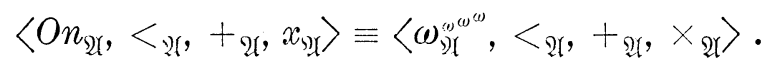

Proof. [7].

Let $\mathfrak{X}$ be an arbitrary $\omega$-model of $Z F$, i.e., an arbitrary model of $Z F$ such that $\omega_{\mathfrak{M}}=\omega$. One can readily show that

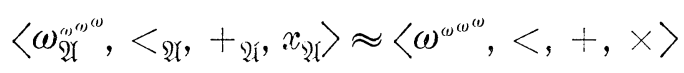

which has a $\Delta_{1}$-truth set. On the other hand,

$$
T h_{\mathfrak{X}} \leqq_{T} \operatorname{Tr}_{\left\langle 0 n_{\mathfrak{W}},<_{\mathfrak{U}}, P R_{\mathfrak{H}}\right\rangle}(<,+, \times, \tilde{\varepsilon})
$$

and $T h_{\mathfrak{H}}$ is not $\Delta_{1}^{1}$ (for any $\omega$-model of $Z F$ includes a definable $\omega$ model of analysis whose truth set is well-known to be not $\Delta_{1}^{1}$ ).

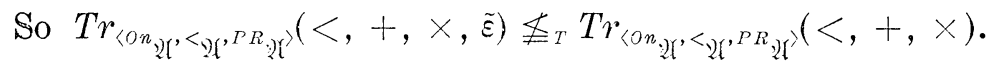

Corollary 8. If $Z F$ has $\omega$-models there are models $\mathfrak{N}_{1}, \mathfrak{U}_{2} \models Z F$

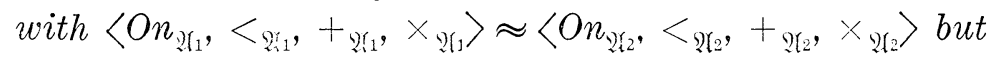

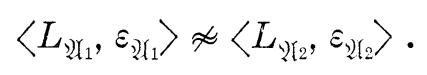

Further extensions of these results involve the use of non primitive recursive functions. So we now define arithmetic functions and give certain elementary properties of them that are needed. A more detailed exposition, including the proofs of the elementary properties is presented in our dissertation [14].

A predicate $A\left(a_{1} \cdots a_{n}\right)$ of ONT is called arithmetic if and only if there is a primitive recursive predicate $B\left(a_{1} \cdots a_{n} x_{1} \cdots x_{m}\right)$ and quantifiers $Q_{1} \cdots Q_{m}$ such that

$$
\left.\right|_{\overline{\sigma_{N T}}}\left(A\left(a_{1} \cdots a_{n}\right) \leftrightarrow\left(Q_{1} x_{1}\right) \cdots\left(Q_{m} x_{m}\right) B\left(a_{1} \cdots a_{n} x_{1} \cdots x_{m}\right)\right) .
$$

The constant, relation and function symbols of $\mathrm{ONT}^{\prime}$ (theory of arithmetic functions on the ordinals) are those of ONT and the arithmetic function symbols described below. For each arithmetic predicate 
$A\left(a_{1} \cdots a_{n+1}\right)$ such that $\left.\right|_{\overline{o N T}}\left(\exists ! a_{n+1}\right) A\left(a_{1} \cdots a_{n+1}\right)$ we introduce a function symbol $f_{A}$ whose intended meaning is to be the function defined by $f_{A}\left(a_{1} \cdots a_{n}\right)=a_{n+1}$ if and only if $A\left(a_{1} \cdots a_{n+1}\right)$.

The axioms of $\mathrm{ONT}^{\prime}$ are those of ONT allowing, however, in all schema arithmetic function symbols together with the "definitions" of the arithmetic function symbols, i.e., if $f_{A}$ is an arithmetic function such that $\mid \overline{O N T}\left(\exists ! a_{n+1}\right) A\left(a_{1} \cdots a_{n+1}\right)$, then

$$
f_{A}\left(a_{1} \cdots a_{n}\right)=a_{n+1} \leftrightarrow A\left(a_{1} \cdots a_{n+1}\right)
$$

is an axiom of $\mathrm{ONT}^{\prime}$.

A predicate $A\left(a_{1} \cdots a_{n}\right)$ of $\mathrm{ONT}^{\prime}$ is called arithmetic if there is an arithmetic predicate $B\left(a_{1} \cdots a_{n}\right)$ of ONT such that

$$
\left.\right|_{\widehat{O N T}}\left(A\left(a_{1} \cdots a_{n}\right) \leftrightarrow B\left(a_{1} \cdots a_{n}\right)\right) .
$$

A predicate $A\left(a_{1} \cdots a_{n+1}\right)$ of $\mathrm{ONT}^{\prime}$ is called a function if

$$
\left.\right|_{\overline{O N T^{\prime}}}\left(\exists ! a_{n+1}\right) A\left(a_{1} \cdots a_{n+1}\right) \text {. }
$$

It is called an arithmetic function if

$$
\mid \overline{\mathrm{ONT}^{\prime}} A\left(a_{1} \cdots a_{n+1}\right) \leftrightarrow f\left(a_{1} \cdots a_{n}\right)=a_{n+1}
$$

for some arithmetic function symbol $f$.

One can readily show using results and techniques of Takeuti for primitive recursive functions.

Lemma 9a. Every primitive resursive function is arithmetic.

Lemma 9b. Every predicate of $\mathrm{ONT}^{\prime}$ is arithmetic, i.e., every predicate consisting only of arithmetic function symbols, constant, relation and function symbols of $\mathrm{ONT}^{\prime},=, \neg, \wedge, \vee, \exists, \forall$ is arithmetic.

Lemma 9c. If $A_{1}(a), \cdots, A_{n}(a)$ are arithmetic predicates such that $\left.\right|_{\overline{O N T}} A_{1}(a) \vee \cdots \vee A_{n}(a)$ and $\left.\right|_{\overline{O N T^{\prime}}} \neg\left(A_{i}(a) \wedge A_{j}(a)\right)$ for $i \neq j$ and if $f_{1}(a) \cdots f_{n}(a)$ are arithmetic functions, then there exists an arithmetic function $f(a)$ such that

$$
\left.\right|_{\overline{O N T^{\prime}}} \bigwedge_{i \leqq n}\left(A_{i}(a) \rightarrow f(a)=f_{i}(a)\right) .
$$

As the arithmetic functions are definable in terms of the primitive recursive predicates and in terms of the primitive recursive functions we have that the results in Lemma 1b, 1c of Takeuti continue to hold, i.e., 
LEMmA 9d. If $\left\langle O n,\langle\right.$, Arith $\rangle \vDash \mathrm{ONT}^{\prime}$, then $\mathfrak{A}=\langle O n, \tilde{\varepsilon}, \cong\rangle \vDash Z F$, $V=L$ and $\left\langle O n,\langle\right.$, Arith $\rangle \approx\left\langle O n_{\mathfrak{H}},<_{\mathfrak{Q}}\right.$, Arith $\left._{\mathfrak{H}}\right\rangle$.

Lemma 9e. If $\mathfrak{A} \vDash Z F$, then $\left\langle O n_{\mathfrak{Q}},<_{\mathfrak{W}}\right.$, Arith $\left.h_{\mathfrak{H}}\right\rangle \vDash O \mathrm{ONT}^{\prime}$. Hence as with ONT we may conclude by use of $\mathrm{ONT}^{\prime}$ that

Lemma 10. Let $A$ be a set of arithmetic functions. If there is a model $\mathfrak{A}$ of $Z F$ and an ordinal $\alpha_{\mathfrak{U}} E O n_{\mathfrak{U}}$ sush that

(1), (2) as in Lemma 5

then the structure of $L$ is not determined up to $\equiv$ by the strusture of the ordinals with the class $A$ of arithmetic functions on it.

The above completes the necessary treatment of arithmetic functions. In the following extensions of Corollary 4, we will encounter cases where although $\left\langle O n_{\mathfrak{U}},<_{\mathfrak{Y}}, A_{\mathfrak{A}}\right\rangle \equiv\left\langle\alpha_{\mathfrak{Q}},<_{\mathfrak{A}}, A_{\mathfrak{U}}\right\rangle$ for an appropriate ordinal $\alpha_{\mathfrak{W}}$, it is not the case that $T h_{\left\langle\alpha_{\mathfrak{X}}{ }^{\prime}{ }_{\mathfrak{H}}, A_{\mathfrak{H}}\right\rangle}$ is $\Delta_{1}^{1}$ or another similarly classified set which we can show $T h_{\mathfrak{A}}$ is not. Hence to handle this situation we put restrictions on the model $\mathfrak{A}$ in the hypothesis of Lemma 10 ; we require $2 \mathbb{l}$ to be a nameable standard model of $Z F$ [10]. (A nameable model is one all of whose sets are definable. If $\widetilde{Z F}$ is a "nice", e.g., finite or recursive, extension of $Z F$, then the minimal standard model of $\widetilde{Z F}$ is nameable.)

Lemma 11. Let $A$ be a set of arithmetic functions.

If there is a nameable standard model $\mathfrak{A}$ of $Z F$ and an ordinal $\alpha_{\mathfrak{U}} \in O n_{\mathfrak{H}}$ such that

1. as in Lemma 10

then the same conclusion as in Lemma 10 holds.

Proof. As $\mathfrak{A}$ is nameable, $T h_{\mathfrak{A}} \notin \mathfrak{U}$ (or else it would be definable contradicting the Tarski theorem on the definability of truth predicates [11]).

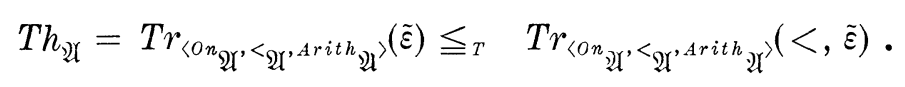

So as standard models are closed under $\leqq_{T}, \operatorname{Tr}_{\left\langle 0 n_{\mathfrak{H}},{ }_{\mathfrak{H}}, A r i t h_{\mathfrak{H}}\right\rangle}(<, \tilde{\varepsilon}) \notin \mathfrak{T}$.

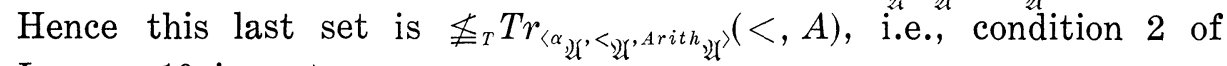
Lemma 10 is met.

The restriction in Lemma 11 to nameable models will often be unessential as we see in Lemma 12 and its applications.

Lemma 12. Let $A$ be a set of arithmetic functions. 
If there is a formula $F$ with 1 free variable such that

(1) there is a standard model $\mathfrak{U}$ of $Z F,(\exists ! x)(F(x) \wedge \operatorname{Ord}(x))$, $V=L($ call this $\widetilde{Z F})$

(2) $\forall n, \widetilde{\widetilde{Z F}}$ Player II has a winning strategy in

$$
H_{n}(\langle O n,\langle, A\rangle,\langle\alpha,\langle, A\rangle)
$$

for $\alpha \in$ On such that $F(\alpha)$, then the strusture of $L$ is not determined up to $\equiv$ by the structure of the ordinals with the class $A$ of arithmetic functions on it.

Proof. As $\widetilde{Z F}$ has a standard model, it has a nameable model B. [10].

So $\mathfrak{B} \vDash(\exists ! x)(F(x) \wedge \operatorname{Ord}(x))$. Let $\beta \approx O n_{\mathfrak{B}}$ such that $\mathfrak{B} \vDash F[\beta]$. By assumption 2, $\mathfrak{B} \vDash$ Player II has a winning strategy in

$$
H_{n}(\langle O n,\langle, A\rangle,\langle\beta,\langle, A\rangle) \text {. }
$$

So condition 1 of Lemma 11 is true in $\mathfrak{B}$.

REMARK. This lemma also holds for finite extensions of $Z F$.

Doner and Tarski (Doner and Tarski 67) define higher exponential functions $O$, as follows:

(1) $\alpha O_{i} \beta=\alpha+\beta$ if $\gamma=0$.

(2) $\alpha O_{\gamma} \beta=\lim _{\eta<\beta, \zeta<\gamma}\left(\left(\alpha O_{i} \eta\right) O_{\zeta} \alpha\right)$ if $\gamma \geqq 1$.

Let $O(\alpha, \beta, \gamma)=\alpha O_{\gamma \beta}$

$$
O^{\delta}(\alpha, \beta, \gamma)=\left\{\begin{array}{l}
O(\alpha, \beta, \gamma) \text { if } \gamma \leqq \delta \\
0 \text { otherwise }
\end{array}\right.
$$

Lemma 13. $O(\alpha, \beta, \gamma)$ is arithmetic.

Proof. We, in fact, illustrate a general method of proving that the arithmetic functions are closed under recursion. The approach is to realize that given $\tilde{\varepsilon}$, $\cong$ we have a model of $Z F$ in $\langle O n$, Arith $\rangle$ in which we can by suitable means define the higher exponential functions on ordinals of this model of set theory. Then by means of the isomorphism of

$$
\left\langle O n_{\langle 0 n, \tilde{\varepsilon},=\rangle}, \tilde{\varepsilon}, \operatorname{Arith}_{\langle 0 n, \tilde{\varepsilon},=\rangle}\right\rangle \underset{\rightrightarrows}{\approx}\langle O n,\langle\text {, Arith }\rangle
$$

we can convert these into the functions on $O n$.

First we give several predicates needed in the discussion of $\angle O n$, $\tilde{\varepsilon}, \cong$ and the map from $O n_{\langle 0 n, \tilde{\varepsilon}, \Rightarrow}$ to $O n$. The reader may readily confirm that these are all arithmetic (usually by the use of some of the previous ones): 
$M(x)=_{d f} \neg\left(\exists \alpha^{\prime}\right)_{<\alpha}\left(\alpha^{\prime} \cong \alpha\right) . \quad(\alpha$ is a set of $\langle O n, \tilde{\varepsilon}, \cong\rangle$. We, thus, successfully isolate a singll representative for each member of the model $\langle O n, \tilde{\varepsilon}, \cong$ of $Z F$. We let $M$ denote $\{x \varepsilon O n \mid M(x)\}$. In particular $\cong \cap M \times M$ is $=$ ).

$\varepsilon^{*}={ }_{d f} \in \cap M \times M,={ }^{*}={ }_{d f} \cong \cap M \times M(=$ ordinary $=)$. $U P(x)={ }_{d f} x$ is an unordered pair in sense of $\left\langle M, \varepsilon^{*},=^{*}\right\rangle$. $x=\{y, z\}^{*}={ }_{d f} x=\{y, z\}$ in sense of $\left.M, \varepsilon^{*},=^{*}\right\rangle$. $O P_{2}(x)={ }_{d f} x$ is an ordered pair in sense of $\left\langle M, \varepsilon^{*},=^{*}\right\rangle$. $x=\langle y, z\rangle^{*}=_{d f} x=\langle y, z\rangle$ in sense of $\left\langle M, \varepsilon^{*},=^{*}\right\rangle$. $O P_{n}(x)={ }_{d f} x$ is an ordered $n$-tuple in sense of $\left\langle M, \varepsilon^{*},=*\right\rangle$. $x=\left\langle x_{1} \cdots x_{n}\right\rangle^{*}=_{d f} x=\left\langle x_{1} \cdots x_{n}\right\rangle$ in sense of $\left\langle M, \varepsilon^{*},={ }^{*}\right\rangle$. $\operatorname{Rel}_{n}(x)=_{d f} x$ is a $n$-ary relation in sense of $\left\langle M, \varepsilon^{*},={ }^{*}\right\rangle$. $F c n_{n}(x)=_{d f} x$ is a $n$-ary function in sense of $\left\langle M, \varepsilon^{*},=^{*}\right\rangle$. $x=f\left(y_{1} \cdots y_{n}\right)^{*}={ }_{d f} x=f\left(y_{1} \cdots y_{n}\right)$ in sense of $\left\langle M, \varepsilon^{*},=^{*}\right\rangle$. The following is primitive recursive

$$
\begin{aligned}
& \operatorname{Ord}(x)={ }_{d f} M(x) \wedge(\forall y)_{<x}(\forall z)_{<x}\left(y \varepsilon^{*} x \wedge z \varepsilon^{*} x\right. \\
& \left.\rightarrow\left(y \varepsilon^{*} z \vee z \varepsilon^{*} y \vee z=y\right)\right) \wedge(\forall y)_{<x}(\forall z)_{<x} \\
& \left(y \varepsilon^{*} z \vee z \varepsilon^{*} y \rightarrow z \varepsilon^{*} x\right)
\end{aligned}
$$

i.e., $\operatorname{Ord}(x)$ if and only if $x$ is an ordinal of $\left\langle M, \varepsilon^{*},=^{*}\right\rangle$ in sense of $\left\langle M, \varepsilon^{*},=^{*}\right\rangle$. As a result by a standard application of primitive recursion $h(x)$ defined on $\left\langle M, \varepsilon^{*},=^{*}\right\rangle$ ordinals as follows is primitive recursive:

$$
h(x)=(\mu \alpha)_{<x^{\prime}}\left((\forall y)\left(y^{*} \varepsilon x \rightarrow h(y)<\alpha\right)\right) .
$$

That is $h$ is the map of $\left\langle M, \varepsilon^{*},=^{*}\right\rangle$-ordinals isomorphically to $O n$.

Hence as + on $O n$ is primitive recursive, + for $\left\langle M, \varepsilon^{*},=^{*}\right\rangle$ is also primitive recursive. We denote it as $+^{*}$. So let $\operatorname{Exp}(f)$ be the predicate expressing that $f$ is an initial piece of higher exponential functions on $\left\langle M, \varepsilon^{*},={ }^{*}\right\rangle$ with all needed induction information to compute any of $f^{\prime}$ s values, i.e., let $\operatorname{Exp}(f)={ }_{d f}$

$\mathrm{Fcn}_{3}(f)-f$ is a 3 -ary function

$\wedge(\forall z)_{\text {ord }(z)}(\forall x)_{\text {Ord }(x)}(\forall y)_{\text {ord }(y)}\left((\exists w)_{\text {ord }(w)}\right.$

$\left(w=f(x, y, z)^{*}\right) \rightarrow\left(\forall y^{\prime}\right)_{\varepsilon^{*} y}\left(\forall z^{\prime}\right)_{z^{*} z}\left(\exists w^{\prime}\right)_{\text {ord }\left(w^{\prime}\right)}\left(\exists w^{\prime \prime}\right)_{\text {ord }\left(w^{\prime \prime}\right)}$

$\left.\left(w^{\prime}=f\left(x, y^{\prime}, z\right)^{*} \wedge w^{\prime \prime}=f\left(w^{\prime}, x, z^{\prime}\right)^{*}\right)\right)$ - initial segment clause

$\wedge(\forall x)(\forall y) \forall z)(\forall w)\left(w=f(x, y, z)^{*} \rightarrow \operatorname{Ord}(x) \wedge \operatorname{Ord}(y)\right.$

$\wedge \operatorname{Ord}(z) \wedge \operatorname{Ord}(w))$ - range and domains in ordinals

$\wedge(\forall x)_{\text {ord }(x)}(\forall y)_{\text {ord }(y)}(\exists w)\left(w=f(x, y, 0)^{*} \rightarrow w=x+{ }^{*} y\right)$

for $z=0,(x, y, z)=x+y$ 


$$
\begin{aligned}
& \wedge(\forall x)_{\operatorname{ord}(x)}(\forall y)_{\operatorname{ord}(y)}(\forall z)_{\operatorname{ord}(z) \wedge \neq 0}(\forall w)\left(w=f(x, y, z)^{*}\right. \\
& \rightarrow\left(\left(\forall z^{\prime}\right)_{\varepsilon^{*} z}\left(\forall y^{\prime}\right)_{\varepsilon^{*} y}\left(f\left(f\left(x, y^{\prime}, z\right)^{*}, x, z^{\prime}\right)^{*} \varepsilon^{*} w \vee=w\right)\right. \\
& \left.\left.\wedge \neg\left(\exists w^{\prime}\right)_{\varepsilon^{*} w}\left(\forall z^{\prime}\right)_{\varepsilon^{*} z}\left(\forall y^{\prime}\right)_{\varepsilon^{*} y}\left(f\left(f\left(x, y^{\prime}, z\right)^{*}, x, z^{\prime}\right)^{*} \varepsilon^{*} w^{\prime} \vee=w^{\prime}\right)\right)\right) \\
& \text { for } z \neq 0, f(x, y, z)=\lim _{y^{\prime}<y, z^{\prime}<z} f\left(f\left(x, y^{\prime}, z\right), x, z^{\prime}\right) .
\end{aligned}
$$

Clearly this is arithmetic.

So $O(\alpha, \beta, \gamma)=\delta \leftrightarrow(\exists f)(\exists x)(\exists y)(\exists z)(\exists w)(\operatorname{Exp}(f) \wedge h(x)=\alpha \wedge h(y)=$ $\left.\beta \wedge h(z)=\gamma \wedge h(w)=\delta \wedge w=f(x, y, z)^{*}\right)$ is arithmetic.

Hence by Lemma $9 \mathrm{c}, O^{s}$ is arithmetic if $\delta$ is definable.

THEOREM 14. [5]. $\left\langle O n,\left\langle, O^{i}\right\rangle \equiv\left\langle\mu\left(\omega^{\omega}, O_{\gamma}\right),\left\langle, O^{i}\right\rangle\right.\right.$

Proof. [5]. $\left(\mu\left(\alpha, O_{i}\right)\right.$ is defined as

$$
\begin{aligned}
& \mu\left(0, O_{\gamma}\right)=0 \\
& \mu\left(\alpha+1, O_{\gamma}\right)=(\mu \beta)_{>\mu\left(\alpha, O_{\gamma}\right)}\left(\forall \delta_{1}\right)\left(\forall \delta_{2}\right)\left(\delta_{1}, \delta_{2}<\beta \rightarrow O_{\gamma}\left(\delta_{1}, \delta_{2}\right)<\beta\right) \\
& \mu\left(\lambda, O_{\gamma}\right)=\lim _{\alpha<\gamma} \mu\left(\alpha, O_{\gamma}\right),
\end{aligned}
$$

i.e., $\mu\left(\alpha, O_{\gamma}\right)$ is the $\alpha$-th critical (or main) value of $O_{\gamma}$ ).

COROLlary 15. Say there is a standard model of

$$
Z F,(\exists ! x)(F(x) \wedge \operatorname{Ord}(x)), V=L .
$$

Then there are models $\mathfrak{U}_{1}, \mathfrak{U}_{2} \vDash Z F,(\exists ! x)(F(x) \wedge \operatorname{Ord}(x))$, and $a_{i} \in \mathfrak{A}_{i}$ such that:

(i ) $\mathfrak{A}_{i} \vDash F\left(a_{i}\right) \wedge \operatorname{Ord}\left(a_{i}\right)$.

(ii) $\left\langle O n_{\mathfrak{U}_{1}},<_{\mathfrak{U}_{1}}, O_{\zeta}^{\mathfrak{\mathscr { U } _ { 1 }}}\right\rangle_{\zeta \leqq a_{1}} \approx\left\langle O n_{\mathfrak{U}_{2}},<_{\mathfrak{U}_{2}}, O_{\zeta}^{\mathfrak{R}_{2}}\right\rangle_{\zeta \leqq a_{2}}$.

(iii) $\left\langle L_{\mathfrak{A}_{1}}, \varepsilon_{\mathfrak{A}_{1}}\right\rangle \approx\left\langle L_{\mathfrak{A}_{2}}, \varepsilon_{\mathfrak{A}_{2}}\right\rangle$.

Proof. Let $Z F^{*}=Z F \cup\{(\exists ! x)(F(x) \wedge \operatorname{Ord}(x))\}$. We reason as follows in $Z F^{*} \cup\{V=L\}$.

Let $\alpha$ be the unique ordinal satisfying $F$. So there is a unique ordinal $\beta$ satisfying the predicate $x=\mu\left(\omega^{\omega}, O_{\alpha}\right)$, i.e., satisfying the predicate $(\exists \gamma)\left(F(\gamma) \wedge x=\mu\left(\omega^{\omega}, O_{\gamma}\right)\right)=G(x)$.

Furthermore, given $n$-Player II has a winning strategy in

$$
H_{n}\left(\left\langle O n,\left\langle, O^{\alpha}\right\rangle,\left\langle\mu\left(\omega^{\omega}, O_{\alpha}\right),<, O^{\alpha}\right\rangle\right)\right.
$$

by proof of Theorem 14 .

So $\left.\right|_{\overline{Z F^{*} \cup\{V=L\}}}(\exists ! x)(G(x) \wedge \operatorname{Ord}(x))$. So if 


$$
\widetilde{Z F}=Z F^{*} \cup\{V=L, \exists ! x(G(x) \wedge \operatorname{Ord}(x))\}
$$

then $\widetilde{Z F}$ has a standard model.

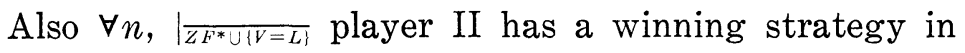

$$
H_{n}\left(\left\langle O n,<, O^{\alpha}\right\rangle,\left\langle\mu\left(\omega^{\omega}, O_{\alpha}\right),\left\langle, O^{\alpha}\right\rangle\right)\right.
$$

where $F(\alpha)$ and $\operatorname{Ord}(\alpha)$.

So $\forall n, \overline{\widetilde{Z F}}$, this fact. These are the hypotheses of Lemma 12 .

REMARK. Corollary 15 may be improved for $O_{\zeta}, \zeta<\gamma$ where $\gamma$ is a recursive ordinal. (The subsequent notation and results in recursion theory to be used in this remark all appear in [13].) In that event we may weaken the hypothesis of Corollary 15 to the assumption of the existence of an $\omega$-model of $Z F$.

As $\gamma$ is recursive, there exists $<_{R}$, a recursive linear order orderisomorphic to $<\uparrow \alpha$. In particular as $<_{R}$ is recursive, $<_{R}^{\mathfrak{N}}=<_{R}$ for every $\omega$-model $\mathfrak{A}$ of analysis and hence of set theory. Let

$$
\left.F(x)=\left(<\uparrow x \approx<_{R}\right) \wedge \operatorname{Ord}(x)\right) .
$$

Let $\mathfrak{A}$ be an $\omega$-model of $Z F$. So $\mathfrak{A} \vDash(\exists ! x) F(x)$. Say $A \vDash F[a]$ (asi). By Theorem 14,

$$
\left\langle O n_{\mathfrak{A}},<_{\mathfrak{A}}, O_{\mathfrak{Q}}^{a}\right\rangle \equiv\left\langle\mu\left(\omega^{\omega}, O_{a, \mathfrak{Q}}\right),<_{\mathfrak{Q}}, O_{\mathfrak{Q}}^{a}\right\rangle .
$$

Now $\mu\left(\omega^{\omega}, O_{a, \mathfrak{Y}}\right)$ is itself a recursive ordinal. This is best seen by using Doner and Tarski's result that

$$
\mu\left(\omega^{\omega}, O_{2 \gamma}\right)=\mu\left(\omega^{\omega}, O_{2 \gamma+1}\right)=\omega O_{2 \gamma+2} \omega^{\omega} .
$$

Hence to show that $\mu\left(\omega^{\omega}, O_{\gamma}\right)$ is recursive, it suffices to show that $\alpha O_{\gamma} \beta$ is recursive for $\alpha, \beta, \gamma$ recursive. This is best done by defining $O(\alpha, \beta, \gamma)$ on $O$, the universal system of notations.

Let $g(a, x, y, z)=$

$$
\begin{array}{ll}
x+{ }_{o} y & \text { if } z=1 \\
1 & \text { if } z \neq 1, y=1 \\
x & \text { if } z \neq 1, y=2 \\
\varphi_{a}\left(\varphi_{a}(x, v, z), x, w\right) & \text { if } z=2^{w}, y=2^{v} \\
3 \cdot 5^{e_{1}} & \text { if } z=3 \cdot 5^{e}, y=2^{v}
\end{array}
$$

$$
\text { where } \varphi_{e_{1}}=(\lambda n)\left(\varphi_{a}\left(\varphi_{a}(x, v, z), x, \varphi_{e}(n)\right)\right.
$$

$3 \cdot 5^{e_{2}}$

if $y=3 \cdot 5^{e}$

$$
\text { where } \varphi_{e_{2}}=(\lambda n)\left(\varphi_{a}\left(x, \varphi_{e}(n), y\right)\right)
$$

Otherwise. 
So $g$ is recursive. So there is a recursive function $h$ such that

$$
g(a, x, y, z)=\varphi_{h\langle a\rangle}(x, y, z) .
$$

By the recursion theorem there is an $n$ such that $\varphi_{n}=\varphi_{h(n)}$. Let $f=\varphi_{n}$.

So $f(x, y, z)=x+{ }_{o} y$

if $z=1$

1

if $z \neq 1, y=1$

$x$

if $z \neq 1, y=2$

$f(f(x, v, z), x, w)$

if $z=2^{w}, y=2^{v}$

$3 \cdot 5^{e_{1}}$

if $z=3 \cdot 5^{e}, y=2^{v}$

where $\varphi_{e_{1}}=(\lambda n)\left(f(x, v, z), x, \varphi_{e}(n)\right)$

$3 \cdot 5^{e_{2}}$

if $y=3 \cdot 5^{e}$

where $\varphi_{e_{2}}=(\lambda n)\left(f\left(x, \varphi_{e}(n), y\right)\right)$

0

Otherwise.

By another theorem of Doner and Tarski we have, $\alpha O_{0} \beta=\alpha+\beta$

$\alpha O_{\gamma} 0=0$

if $\gamma \geqq 1$

$\alpha O_{\gamma} 1=\alpha$

if $\gamma \geqq 1$

$\alpha O_{\gamma+1}(\beta+1)=\left(\alpha O_{\gamma+1} \beta\right) O_{\gamma} \alpha$

$\alpha O_{\lambda}(\beta+1)=\lim _{\eta<\lambda}\left(\alpha O_{\lambda} \beta\right) O_{r} \alpha$

if $\lim (\lambda)$

$\alpha O_{r} \lambda=\lim _{\beta<\lambda}\left(\alpha O_{r} \beta\right)$

if $\lim (\lambda)$.

So by induction on $O$, we have if $x, y, z \in O$, then

$$
|f(x, y, z)|_{o}=|x|_{o} O_{|z|}|y|_{o} \text { and } f(x, y, z) \in O .
$$

So if $\alpha, \beta, \gamma$ are recursive ordinals, then $\alpha O_{\gamma} \beta$ is a recursive ordinal. In particular $\mu\left(\omega^{\omega}, O_{a, \mathfrak{U}}\right)$ is a recursive ordinal. Let $<_{s}$ be a recursive linear ordering such that $<_{\mathfrak{A}} \uparrow O_{a}, \mathfrak{A} \approx<_{s}$. Then define $O_{\mathfrak{U}}^{a}$ according to $<_{s}$ by induction on $<_{s}$. It has an arithmetic definition. So as

$$
\left\langle\mu\left(\omega^{\omega}, O_{a, \mathfrak{I}}\right),<_{\mathfrak{Q}}, O_{\mathfrak{V}}^{a}\right\rangle \approx\left\langle\operatorname{Dom}\left(<_{s}\right),<_{s}, O_{\mathfrak{U}}^{a} \text { according to }<_{s}\right\rangle,
$$

$\left\langle O n_{\mathfrak{Y}},<_{\mathfrak{Q}}, O_{\mathfrak{A}}^{\mathfrak{l}}\right\rangle \equiv\left\langle\operatorname{Dom}\left(<_{s}\right),<_{s}, O_{\mathfrak{A}}^{\mathfrak{a}}\right.$ according to $\left.<_{s}\right\rangle$ which has a $\Delta_{1}^{1}$ truth-set as Dom $\left(<_{s}\right),<_{s}, O_{\mathfrak{Q}}^{a}$ according to $<_{s}$ are all arithmetic.

On the other hand, as $\mathfrak{U}$ is an $\omega$-model of set theory, $P(\omega)_{\mathfrak{A}}$ is an $\omega$-model of analysis and $T h_{P(\omega)} \leqq_{T} T h_{\mathfrak{Q}} \cdot$ But the former is not $\Delta_{1}^{1}$ and so neither is $T h_{\mathfrak{Z}}$ or $\operatorname{Tr}_{\left\langle o n_{\mathfrak{H}},{ }_{\mathfrak{Y}},{ }^{\mathrm{Arith}} \mathfrak{\mathscr { A }}\right\rangle}\left(<, O^{a}, \tilde{\varepsilon}\right)$. So as in the proof of Corollary 8 , if there is an $\omega$-model $\mathfrak{A}$ of set theory, then there are models $\mathfrak{A}_{1}, \mathfrak{A}_{2} \vDash Z F, \exists ! x F(x)$ and ordinals $a_{i} \in \mathfrak{A}_{i}$ such that

(i) $\quad \mathfrak{V}_{i} \vDash F\left[a_{i}\right]$

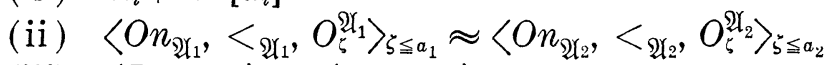

(iii) $\left\langle L_{\mathfrak{A}_{1}}, \varepsilon_{\mathfrak{Q}_{1}}\right\rangle \neq\left\langle L_{\mathfrak{Q}_{2}}, \varepsilon_{\mathfrak{A}_{2}}\right\rangle$ 
(provided that in any $\omega$-model, the unique ordinal $\alpha$ such that $F(\alpha)$ is recursive).

Let $\gamma$ be a definable ordinal. Given an arithmetic function $f$ we define $f_{\curlyvee}$ as follows:

$$
f_{i}\left(\alpha_{1} \cdots \alpha_{n}\right)=\left\{\begin{array}{l}
f\left(\alpha_{1} \cdots \alpha_{n}\right) \text { if } \alpha_{1} \cdots \alpha_{n}, f\left(\alpha_{1} \cdots \alpha_{n}\right)<\gamma \\
0 \text { otherwise. }
\end{array}\right.
$$

So by Lemma $9 \mathrm{c}$, each $f_{\gamma}$ is arithmetic.

Let $A$ rith $h_{\gamma}=\left\{f_{\gamma} \mid f \in\right.$ Arith $\}$.

We now proceed to show that appropriate ordinals can be found so that we can show $\left\langle\alpha,\left\langle, A^{\prime}, A r i t h_{i}\right\rangle \equiv\left\langle O n,\left\langle, A^{\prime}, A r i t h_{i}\right\rangle\right.\right.$ for various $A^{\prime} \subseteq$ Arith. Once if we have shown this (provided that $\alpha$ is provably less than $O n$, so that it is less than $O n$ in all models) we immediately can obtain not determined up to $\equiv$ results as in Corollary 15 above.

In order to obtain such appropriate $\alpha$ we must briefly consider $H_{n}$ in greater detail. $H_{n}$ is an $n$ move finite 2 person game. At move $i$, player 1 choses a model $\mathfrak{A}_{l(i)}$, an integer $k(i)$ and $k(i)$ points $a_{1 i}^{l(i)} \cdots a_{k(i, i}^{l(i)}$ in model $\mathfrak{A}_{l(i)}$. Player 2 responds by choosing $k(i)$ points $a_{k k(i) i}^{3-l i}$ in the other model. At the end Player 2 is said to win just in case the correspondence $a_{j i}^{1} \leftrightarrow a_{j i}^{2} \mathrm{i}=1 \cdots n, j=1 \cdots k(i)$ is a partial isomorphism (with respect to the relations and operations of $\mathfrak{A}_{1}$ and $\mathfrak{A}_{2}$ ).

So in a game $H_{n}\left(\left\langle\alpha,\left\langle, A^{\prime}\right\rangle,\left\langle O n,\left\langle, A^{\prime}\right\rangle\right)\right.\right.$ if 2's winning strategy can be constructed to preserve ordinals $<\gamma$, then it is clear that the partial isomorphism will also extend to Arith ${ }_{*}$.

Lemma 16. Player 2 has a winning strategy in $H_{n}\left(\left\langle\mu\left(\gamma+\omega^{\omega}\right),<\right\rangle\right)$ preserving ordinals $<\gamma$.

Proof. 2's strategy is as follows:

For ordinals $<\gamma$ he leaves them fixed. For the $\omega^{\omega}$ segment between $\gamma$ and $\gamma+\omega^{\omega}$ "versus" the $O n$ segment $\geqq \gamma, 2$ uses his winning strategy from $H_{n}\left(\left\langle\omega^{\omega},\langle\rangle,\langle O n,\langle\rangle)\right.\right.$ "shifted over by $\gamma$ ".

Let $\gamma^{*}=\lim _{\substack{n<\omega \\ \alpha<i}} \omega^{\alpha} \cdot n$. Then clearly $\gamma^{*} \geqq \gamma($ for $\gamma>0)$.

Lemma 17. Player 2 has a winning strategy in

$$
H_{n}\left(\left\langle\omega^{\gamma+\omega^{\omega}},\langle,+\rangle,\langle O n,<,+\rangle\right)\right.
$$

preserving ordinals $<\gamma^{*}$.

Proof. One simply observes that in the winning strategy for 2 
defined by Ehrenfeucht in this game from the winning strategy for 2 in the game $H_{n}\left(\left\langle\gamma+\omega^{\omega},\langle\rangle,\langle O n,\langle\rangle)\right.\right.$ if ordinals $\langle\gamma$ are preserved in the latter game, then ordinals $<\gamma^{*}$ are preserved in the former.

Lemma 18. Player 2 has a winning strategy in

$$
H_{n}\left(\left\langle\omega^{\omega \gamma+\omega^{\omega}},\langle,+, \times\rangle,\langle O n,\langle,+, \times\rangle)\right.\right.
$$

preserving ordinals $<\gamma^{* *}$.

Proof. As in Lemma 17.

THEOREM 19.

$$
\left\langle\omega^{\omega \tilde{\gamma}+\omega^{\omega}},<,+, \times, A r i t h_{\gamma}\right\rangle \equiv\left\langle O n,<+, \times, A r i t h_{\gamma}\right\rangle .
$$

Proof. As described above.

COROLlary 20. Say there is a standard model of

$$
Z F,(\exists ! x)(F(x) \wedge \operatorname{Ord}(x)), V=L .
$$

Then there are models $\mathfrak{A}_{1}, \mathfrak{A}_{2} \vDash Z F, a_{i} \in A_{i}$ such that

(i) $\mathfrak{A}_{i} \vDash F\left(a_{i}\right) \wedge \operatorname{Ord}\left(a_{i}\right)$

(ii) $\left\langle O n_{\mathfrak{Q}_{1}},<_{\mathfrak{U}_{1}},+_{\mathfrak{U}_{1}}, \times_{\mathfrak{Q}_{1}}, \operatorname{Arith}_{a_{1}}^{\mathfrak{R}_{1}}\right\rangle$

(iii) $L_{\mathfrak{A}_{1}} \not \equiv L_{\mathfrak{Q}_{2}}$. $\approx\left\langle O n_{\mathfrak{U}_{2}},<_{\mathfrak{H}_{2}},+_{\mathfrak{H}_{2}}, \times_{\mathfrak{A}_{2}}, \operatorname{Arith}_{a_{2}}^{\mathfrak{R}_{2}}\right\rangle$

Proof. As in Corollary 15.

REMARKS. (1) If $F(x)$ is a predicate of the form $<\uparrow x \approx<_{R}$ for some recursive well-ordering $<_{R}$, then the hypothesis of corollary 20 may be weakened as were those of corollary 15 to supposing the existence of $\omega$-models of $Z F$.

(2) Doner claims that Lemma 18 may be extended to the statement that 2 has a winning strategy in

$$
H_{n}\left(\left\langle\mu\left(\delta+\omega^{\omega}, O^{r}\right),\left\langle, O^{r}\right\rangle,\left\langle O n,\left\langle, O^{r}\right\rangle\right)\right.\right.
$$

preserving ordinals $<\delta$. Hence we may conclude by similar arguments to those above that the constructible sets are not determined up to $\equiv$ by $<$, Arith $_{\hat{\delta}}$, $O^{r}$ where $\delta, \gamma$ are definable ordinals. Again, as usual the hypothesis may be weakened if both $\delta, \gamma$ are recursive ordinals.

(3) The pairing function $p$ of Godel [8] can be shown by a long tedious computation to be definable in terms of,$+ \times$. Hence in any result where we have shown that the structure of $L$ is not de- 
termined up to $\equiv$ by $<, A^{\prime}$ where,$+ \times \varepsilon A^{\prime}$ we may conclude that the structure of $L$ is not determined up to $\equiv$ by $<, A^{\prime} \cup\{p\}$. Feferman has pointed out to us that the pairing function $q(\alpha, \beta)=2^{\alpha}(\beta+1)$ is immediately definable in terms of,$+ \times$ and exponentiation $\left(O_{2}\right)$, and hence if the structure of $L$ is not determined up to $\equiv$ by $<, A^{\prime}$ where $O_{0}, O_{1}, O_{2} \varepsilon A^{\prime}$ then $A^{\prime}$ might as well contain $q$.

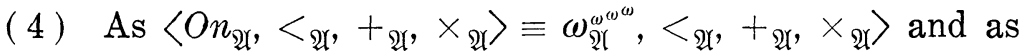

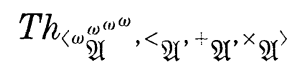

is not recursive for any model $\mathfrak{A}$ of $Z F$, we may conclude that

$$
\operatorname{Tr}_{\langle 0 n,<\cdot A r i t h\rangle}(<,+, \times) \geqq_{T} \operatorname{Tr}_{\langle o n,<, A r i t h\rangle}(<) .
$$

Hence if $Z F$ is consistent, then there are models $\mathfrak{A}_{1}, \mathfrak{A}_{2}$ of $Z F$ with $\left\langle O n_{\mathfrak{Y}_{1}},<_{\mathfrak{A}_{1}}\right\rangle \approx\left\langle O n_{\mathfrak{Y}_{2}},<_{\mathfrak{A}_{2}}\right\rangle$ but

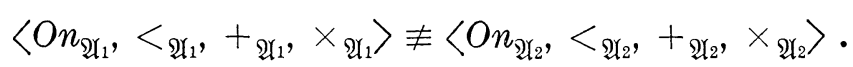

Identically we may conclude that if $Z F$ is consistent then $+{ }^{\omega}, x^{\omega}$ are not determined up to $\equiv$ by $<$ where

$$
\begin{aligned}
& x^{\omega}(\alpha, \beta)=\left\{\begin{array}{l}
\alpha+\beta \text { if } \alpha, \beta<\omega \\
0 \text { otherwise }
\end{array}\right. \\
& x^{\omega}(\alpha, \beta)=\left\{\begin{array}{l}
\alpha \cdot \beta \text { if } \alpha, \beta<\omega \\
0 \text { otherwise }
\end{array}\right.
\end{aligned}
$$

and hence likewise the structure of $L$ is not determined up to $\equiv$ by $<,+^{\omega}, x^{\omega}$ (if $Z F$ has $\omega$-models). Now let HFS be the hereditarily finite sets. Then it is easily seen that there is a one-to-one onto definable map of HFS into $\omega$ such that the image of $\varepsilon$ and $=$ are arithmetic in the sense of recursion theory and hence in particular definable in terms of $+_{\omega}, \times_{\omega}$. Also $+_{\omega}, \times_{\omega}$ are definable in terms of $\varepsilon$ on HFS simply by the use of their recursive definitions. Hence if $Z F$ is consistent then the structure of HFS is not determined up to $\equiv$ by $<$, and if $Z F$ has $\omega$-models then the structure of $L$ is not determined up to $\equiv$ by the structure of HFS.

(5) On the other hand, as $\tilde{\varepsilon}$ gives us in $\left\langle O n_{\mathfrak{Y}},\left\langle\right.\right.$, Arith $\left.h_{\mathfrak{A}}\right\rangle$ (for any model $\mathfrak{A}$ of $Z F$ ) a model of $Z F$ we may in this model give explicit definitions of all primitive recursive functions which are of course definable in terms of $\tilde{\varepsilon}$ and hence arithmetic and as $h$ the function mapping ordinals of this model isomorphically to $O n_{\mathfrak{A}}$ is also primitive recursive, we can pull these functions over to arithmetic functions on $O n_{\mathfrak{Q}}$. By the axiom schema of transfinite induction in ONT' these are the original primitive recursive functions. So if $i:\left\langle O n_{1},<_{1}, \tilde{\varepsilon}_{1}\right\rangle \approx$

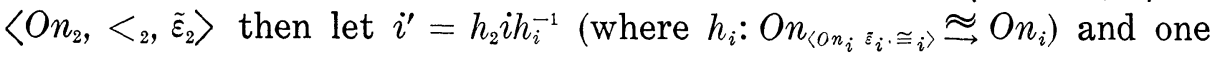


may immediately conclude that $i^{\prime}:\left\langle O n_{1}, A r i t h_{1}\right\rangle \underset{\rightarrow}{\approx}\left\langle O n_{2}, A r i t h_{2}\right\rangle$. So all arithmetic functions are determined up to $\approx$ by $\tilde{\varepsilon}$.

(6) Finally we observe, if we restrict the question if the structure of $L$ is determined up to $\approx$ by $<$ merely to standard models, then the answer, as is well-known, is yes because to show the $L$ 's are isomorphic we have available "real" transfinite induction in the "real" world, i.e., in our metalanguage outside our models.

\section{BIBLIOGRAPHY}

1. Addison, Henkin, and Tarski (eds.), Theory of Models, North Holland, 1965.

2. Every W. Beth, On Padua's Method in the theory of definition, Nederl. Akad. Wetensch. Proc. (A) 56 (1953), 330-339.

3. J. R. Buchi, Relatively categorical and normal theories in Addison, Henkin, Tarski (eds.), The Theory of Models, 65.

4. J. R. Buchi and K. J. Darhof, A strong form of Beth's Definability Theorem, Notices Amer. Math. Soc. 15 (1968), 932.

5. John Doner, Thesis 1968, unpublished, University of California at Berekeley.

6. J. Doner and A. Tarksi, An extended Arithmetic of ordinal numbers, mimeographed notes.

7. A. Ehrenfeucht, An application of games to the completeness problem for formalized theories, Fund. Math. 49 (1961), 129-141.

8. K. Godel, The Consistency of the Continuum Hypothesis, Princeton University Press, 1940.

9. A. Mostowski and A. Tarski, Bull. Amer. Math. Soc. 55 (1949-50), 65.

10. J. Myhill, The hypothesis that all classes are nameable, PNAS 38 (1952).

11. A. Robinson, Introduction to Model Theory and to the Metamathematics of Algebra, North Holland, 1963.

12. J. Robinson, Definability and decision problems in arithmetic, J. Symbolic Logic 14 (1949), 98-114.

13. Hartley Rogers, Jr., Theory of Recursive Functions and Effective Computability, McGraw Hill, 1967.

14. J. Rosenthal, Thesis Part I, 1968, unpublished. M. I. T.

15. Schoenfield, Mathematical Logic, Addison Wesley, 1967.

16. G. Takeuti, A formalization of the theory of ordinal numbers, J. Symbolic Logic 30 (1965).

17. A. Tarski, Der Wahrheitsbeitsbegriff in den formalisierten Sprach, Studia Philosophica 1 (1935), 261-405.

Received July 31, 1969, and in revised form February 10, 1970. Most of the contents of this paper are a portion of the author's Ph. D. thesis (M. I. T. 1968) supervised by Gerald E. Sacks and supported by an N.S. F. Graduate Fellowship. 


\section{PACIFIC JOURNAL OF MATHEMATICS}

\section{EDITORS}

\author{
H. SAMElson \\ Stanford University \\ Stanford, California 94305 \\ C. R. HOBBY \\ University of Washington \\ Seattle, Washington 98105
}

J. DugundjI

Department of Mathematics University of Southern California Los Angeles, California 90007

RICHARD ARENS

University of California

Los Angeles, California 90024

\section{ASSOCIATE EDITORS}
E. F. BECKENBACH
B. H. NeUmanN
F. WOLE
K. YOSHIDA

\section{SUPPORTING INSTITUTIONS}

\author{
UNIVERSITY OF BRITISH COLUMBIA \\ CALIFORNIA INSTITUTE OF TECHNOLOGY \\ UNIVERSITY OF CALIFORNIA \\ MONTANA STATE UNIVERSITY \\ UNIVERSITY OF NEVADA \\ NEW MEXICO STATE UNIVERSITY \\ OREGON STATE UNIVERSITY \\ UNIVERSITY OF OREGON \\ OSAKA UNIVERSITY \\ UNIVERSITY OF SOUTHERN CALIFORNIA
}

\author{
STANFORD UNIVERSITY \\ UNIVERSITY OF TOKYO \\ UNIVERSITY OF UTAH \\ WASHINGTON STATE UNIVERSITY \\ UNIVERSITY OF WASHINGTON \\ * * * \\ AMERICAN MATHEMATICAL SOCIETY \\ CHEVRON RESEARCH CORPORATION \\ NAVAL WEAPONS CENTER
}

The Supporting Institutions listed above contribute to the cost of publication of this Journal, but they are not owners or publishers and have no responsibility for its content or policies.

Mathematical papers intended for publication in the Pacific Journal of Mathematics should be in typed form or offset-reproduced, (not dittoed), double spaced with large margins. Underline Greek letters in red, German in green, and script in blue. The first paragraph or two must be capable of being used separately as a synopsis of the entire paper. The editorial "we" must not be used in the synopsis, and items of the bibliography should not be cited there unless absolutely necessary, in which case they must be identified by author and Journal, rather than by item number. Manuscripts, in duplicate if possible, may be sent to any one of the four editors. Please classify according to the scheme of Math. Rev. Index to Vol. 39. All other communications to the editors should be addressed to the managing editor, Richard Arens, University of California, Los Angeles, California, 90024.

50 reprints are provided free for each article; additional copies may be obtained at cost in multiples of 50 .

The Pacific Journal of Mathematics is published monthly. Effective with Volume 16 the price per volume (3 numbers) is $\$ 8.00$; single issues, $\$ 3.00$. Special price for current issues to individual faculty members of supporting institutions and to individual members of the American Mathematical Society: $\$ 4.00$ per volume; single issues $\$ 1.50$. Back numbers are available.

Subscriptions, orders for back numbers, and changes of address should be sent to Pacific Journal of Mathematics, 103 Highland Boulevard, Berkeley, California, 94708.

PUBLISHED BY PACIFIC JOURNAL OF MATHEMATICS, A NON-PROFIT CORPORATION

Printed at Kokusai Bunken Insatsusha (International Academic Printing Co., Ltd.), 7-17, Fujimi 2-chome, Chiyoda-ku, Tokyo, Japan. 


\section{Pacific Journal of Mathematics}

\section{Vol. 37, No. $2 \quad$ February, 1971}

Charles Compton Alexander, Semi-developable spaces and quotient images of metric spaces .................................... 277

Ram Prakash Bambah and Alan C. Woods, On a problem of Danzer. . . . . . . . . 295

John A. Beekman and Ralph A. Kallman, Gaussian Markov expectations and related integral equations . ....................................

Frank Michael Cholewinski and Deborah Tepper Haimo, Inversion of the Hankel

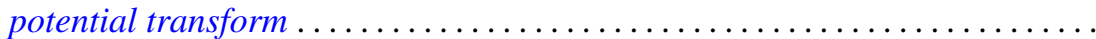

John H. E. Cohn, The diophantine equation

$$
Y(Y+1)(Y+2)(Y+3)=2 X(X+1)(X+2)(X+3) \ldots \ldots \ldots \ldots \ldots
$$

Philip C. Curtis, Jr. and Henrik Stetkaer, A factorization theorem for analytic

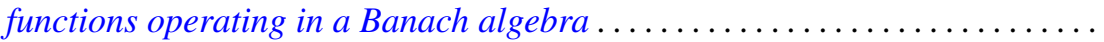

Doyle Otis Cutler and Paul F. Dubois, Generalized final rank for arbitrary limit ordinals

Keith A. Ekblaw, The functions of bounded index as a subspace of a space of entire functions

Dennis Michael Girard, The asymptotic behavior of norms of powers of

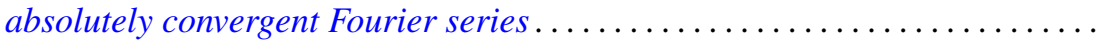

John Gregory, An approximation theory for elliptic quadratic forms on Hilbert spaces: Application to the eigenvalue problem for compact quadratic forms. 383

Paul C. Kainen, Universal coefficient theorems for generalized homology and stable cohomotopy.

Aldo Joram Lazar and James Ronald Retherford, Nuclear spaces, Schauder bases, and Choquet simplexes.

David Lowell Lovelady, Algebraic structure for a set of nonlinear integral operations

John McDonald, Compact convex sets with the equal support property . 429

Forrest Miller, Quasivector topologies

Marion Edward Moore and Arthur Steger, Some results on completability in commutative rings.

A. P. Morse, Taylor's theorem

Richard E. Phillips, Derek J. S. Robinson and James Edward Roseblade, Maximal subgroups and chief factors of certain generalized soluble groups.

Doron Ravdin, On extensions of homeomorphisms to homeomorphisms ...

John William Rosenthal, Relations not determining the structure of $\mathrm{L}$

Prem Lal Sharma, Proximity bases and subbases ........... .

Larry Smith, On ideals in $\Omega_{*}^{u}$. .

Warren R. Wogen, von Neumann algebras generated by operators similar to normal operators

R. Grant Woods, Co-absolutes of remainders of Stone-Čech 